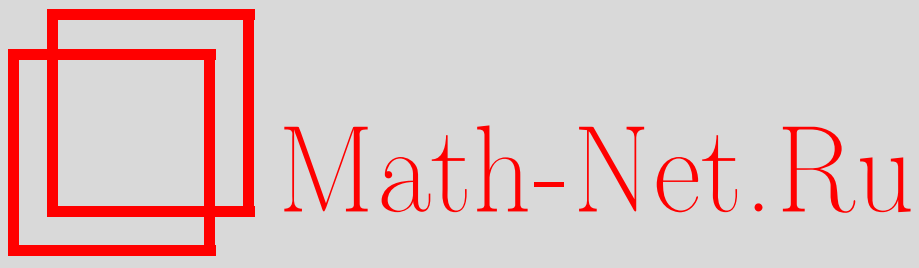

Л. И. Фридман, К. С. Моргачев, Решение стационарной динамической задачи для кольцевой пластины в рамках модели Тимошенко, Вестн. Сам. гос. техн. ун-та. Сер. Физ.-мат. науки, 2005, выпуск 34, 68-71

DOI: https://doi.org/10.14498/vsgtu339

Использование Общероссийского математического портала Math-Net.Ru подразумевает, что вы прочитали и согласны с пользовательским соглашением http://www.mathnet.ru/rus/agreement

Параметры загрузки:

IP : 3.89 .185 .249

26 апреля 2023 г., 13:31:14

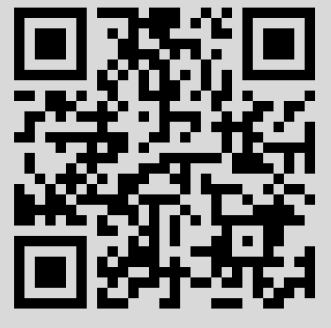


УДК 539.3

Л.И. Фридман, К.С. Моргачев

\section{РЕШЕНИЕ СТАЦИОНАРНОЙ ДИНАМИЧЕСКОЙ ЗАДАЧИ ДЛЯ КОЛЬЦЕВОЙ ПЛАСТИНЫ В РАМКАХ МОДЕЛИ ТИМОШЕНКО}

Получено решение стационарной динамической задачи для кольцевой пластины в рамках модели Тимошенко. Разработанный алгоритм и программы определения частот и форм собственных колебаний, реализованные с использованием средств системы компьютерной математики Матhетатіса, справедливы для любых сочетаний следующих граничных условий на краях пластины: свободный край, шарнирное опирание и жесткая заделка. Достоверность результатов вычисления частот подтверждается численным экспериментом - возбуждением резонансных колебаний.

Введение. Необходимость применения модели Тимошенко при динамических расчетах элементов машин и сооружений, схематизируемых кольцевыми пластинами, обоснована в [1], где приводится сравнение результатов решения динамических задач для цилиндрических тел согласно гипотезе Тимошенко и на основе методов теории упругости с результатами, полученными экспериментально.

В предлагаемой статье на основе известных зависимостей теории колебаний пластин и оболочек построен алгоритм вычисления частот и форм собственных колебаний кольцевой пластины (модель Тимошенко) для любых сочетаний общепринятых граничных условий на ее краях (свободный край, шарнирное опирание и жёсткая заделка).

Для реализации решения использовались широкие возможности системы компьютерной математики Mathematica 4.1, которые позволяют освободить проектировщика от громоздких аналитических преобразований при разработке программ, усовершенствовать процесс вычислений, а также представить результаты вычислений в наглядном виде.

Для подтверждения достоверности результатов вычисления частот была построена программа численного эксперимента: фиксация резонансных колебаний при изменении частоты возбуждающей силы вблизи собственной частоты колебаний пластины (проверка на резонанс).

Основные зависимости для кольцевых пластин (модель Тимошенко). Полученные в [2] разрешающие уравнения колебаний пластины Тимошенко-Уфлянда-Миндлина инвариантны по отношению к системе координат, т.е. будут справедливы и в безразмерных полярных координатах ( $\alpha_{1}=r$ - полярный радиус, отнесенный к внешнему радиусу кольцевой пластины $R$, $\alpha_{2}=\theta$ - полярный угол):

$$
\begin{gathered}
\Delta_{1} F=\frac{2(1+v)}{E k \varepsilon} q, \\
\nabla^{2} \Psi-\frac{2}{1-v}\left(b \Psi+\frac{\partial^{2} \Psi}{\partial t^{2}}\right)=0,
\end{gathered}
$$

где $F(r, \theta)$ и $\Psi(r, \theta)$ - потенциальные функции,

$$
\Delta_{1}=\nabla^{2} \nabla^{2}-\left(1+\frac{2}{k(1-v)}\right) \frac{\partial^{2}}{\partial t^{2}} \nabla^{2}+\frac{2}{k(1-v)} \frac{\partial^{4}}{\partial t^{4}}-\frac{12}{\varepsilon^{2}} \frac{\partial^{2}}{\partial t^{2}},
$$

$\nabla^{2}$ - оператор Лапласа; $q$ - внешняя нагрузка; $b=\frac{6 k(1-v)}{\varepsilon^{2}} ; h$ и $\varepsilon=\frac{h}{R}$ - соответственно абсолютная и относительная толщины пластины; $t$ - безразмерное время, отнесённое к $\frac{R}{c \sqrt{1-v^{2}}}$; $c=\sqrt{\frac{E}{\rho}}$ - скорость звука в материале пластины; $E, v$ и $\rho$ - соответственно модуль упругости, коэффициент Пуассона и массовая плотность материала пластины; $k$ - коэффициент сдвига, определяемый по Миндлину в соответствии с [3] из уравнения:

$$
16\left(1-\frac{1-2 v}{2(1-v)} k\right)(1-k)-(2-k)^{4}=0
$$

Моменты и перерезывающие силы определяются зависимостями 


$$
\begin{gathered}
M_{r}=\frac{D}{R}\left[\frac{\partial \vartheta_{r}}{\partial r}+v\left(\frac{1}{r} \frac{\partial \vartheta_{\theta}}{\partial \theta}+\frac{1}{r} \vartheta_{r}\right)\right] ; M_{\theta}=\frac{D}{R}\left[\frac{1}{r} \frac{\partial \vartheta_{\theta}}{\partial \theta}+\frac{1}{r} \vartheta_{r}+v \frac{\partial \vartheta_{r}}{\partial r}\right] ; \\
M_{r \theta}=\frac{D}{R} \frac{1-v}{2}\left(\frac{1}{r} \frac{\partial \vartheta_{r}}{\partial \theta}+\frac{\partial \vartheta_{\theta}}{\partial r}-\frac{1}{r} \vartheta_{\theta}\right) ; \\
Q_{r}=\frac{k E h}{2(1+v)}\left(\vartheta_{r}-\frac{\partial u}{\partial r}\right) ; Q_{\theta}=\frac{k E h}{2(1+v)}\left(\vartheta_{\theta}-\frac{1}{r} \frac{\partial u}{\partial \theta}\right),
\end{gathered}
$$

где $Q_{r}, M_{r}, M_{r \theta}$-погонная перерезывающая сила, изгибающий и крутящий моменты на линии $r=$ const $Q_{\theta}, M_{\theta}, M_{r \theta}$ - то же, на линии $\theta=$ const $; \vartheta_{r}, \vartheta_{\theta}$ - проекции осредненного поворота на плоскости, образованные нормалью к срединной поверхности и касательной соответственно к линиям $r$ const и $\theta$ const $; u$ - безразмерное перемещение, нормальное к срединной плоскости; $D=\frac{E h^{3}}{12\left(1-v^{2}\right)}$ - цилиндрическая жесткость.

Потенциальные функции $F(r, \theta)$ и $\Psi(r, \theta)$ связаны с $u, \vartheta_{r}$ и $\vartheta_{\theta}$ зависимостями

$$
u=\nabla^{2} F-b F-\frac{\partial^{2} F}{\partial t^{2}} ; \quad \vartheta_{r}=-b \frac{\partial F}{\partial r}-\frac{1}{r} \frac{\partial \Psi}{\partial \theta} ; \vartheta_{\theta}=-b \frac{1}{r} \frac{\partial F}{\partial \theta}+\frac{\partial \Psi}{\partial r} .
$$

При отсутствии нагрузки (стационарная задача) (1) примет вид

$$
\Delta_{1} F=0 .
$$

При колебаниях по $j$-той собственной форме потенциальные функции, перемещения, осредненные повороты, моменты и перерезывающие силы меняются во времени по гармоническому закону (т.е. в обозначениях упомянутых выше величин появится индекс $j$ ).

Оператор $\Delta_{1}$ при определении функции $F_{j}$ из (7) примет вид:

$$
\Delta_{1}=\nabla^{2} \nabla^{2}+\left(1+\frac{2}{k(1-v)}\right) \lambda_{j}^{2} \nabla^{2}+\frac{2}{k(1-v)} \lambda_{j}^{4}-\frac{12}{\varepsilon^{2}} \lambda_{j}^{2}=\left(\nabla^{2}-\alpha_{1 j}^{2}\right)\left(\nabla^{2}+\alpha_{2 j}^{2}\right),
$$

где $\lambda_{j}-$ безразмерная частота колебаний пластины (переход к частотам в герцах осуществляется по формуле $\left.f_{j}=\lambda_{j} \frac{c \sqrt{1-v^{2}}}{2 \pi R}\right)$;

$$
\begin{aligned}
& \alpha_{1 j}^{2}=-\frac{1}{2}\left(1+\frac{2}{k(1-v)}\right) \lambda_{j}^{2}+\sqrt{\frac{1}{4}\left(1-\frac{2}{k(1-v)}\right)^{2} \lambda_{j}^{4}+\frac{12}{\varepsilon^{2}} \lambda_{j}^{2}} ; \\
& \alpha_{2 j}^{2}=\frac{1}{2}\left(1+\frac{2}{k(1-v)}\right) \lambda_{j}^{2}+\sqrt{\frac{1}{4}\left(1-\frac{2}{k(1-v)}\right)^{2} \lambda_{j}^{4}+\frac{12}{\varepsilon^{2}} \lambda_{j}^{2}} .
\end{aligned}
$$

Уравнения (2) и (7) при стационарных колебаниях примут вид

$$
\begin{gathered}
\nabla^{2} \nabla^{2} F_{j}+\left(1+\frac{2}{k(1-v)}\right) \lambda_{j}^{2} \nabla^{2} F_{j}+\frac{2}{k(1-v)} \lambda_{j}^{4} F_{j}-\frac{12}{\varepsilon^{2}} \lambda_{j}^{2} F_{j}=0 ; \\
\nabla^{2} \Psi_{j}-\frac{2}{1-v}\left(b-\lambda_{j}^{2}\right) \Psi_{j}=0 .
\end{gathered}
$$

Функция $F_{j}$ может быть представлена в виде суммы двух функций:

$$
F_{j}=F_{1 j}+F_{2 j} .
$$

Тогда (10) принимают вид уравнений Гельмгольца

$$
\nabla^{2} F_{1 j}-\alpha_{1 j}^{2} F_{1 j}=0 ; \quad \nabla^{2} F_{2 j}+\alpha_{2 j}^{2} F_{2 j}=0 ; \quad \nabla^{2} \Psi_{j}-\beta_{j}^{2} \Psi_{j}=0,
$$

где $\beta_{j}^{2}=\frac{2}{1-v}\left(b-\lambda_{j}^{2}\right)$.

Уравнения (11) решаются методом разделения переменных. С учетом условия периодичности по угловой координате решения уравнений (11) записываются в виде

$$
\begin{gathered}
F_{1 j}=\left(C_{1} I_{m}\left(r \alpha_{1 j}\right)+C_{2} K_{m}\left(r \alpha_{1 j}\right)\right) \cos (m \theta) ; \\
F_{2 j}=\left(C_{3} J_{m}\left(r \alpha_{2 j}\right)+C_{4} Y_{m}\left(r \alpha_{2 j}\right)\right) \cos (m \theta) ; \\
\Psi_{j}=\left(C_{5} I_{m}\left(r \beta_{j}\right)+C_{6} K_{m}\left(r \beta_{j}\right)\right) \sin (m \theta) .
\end{gathered}
$$


Для кольцевых пластин условие периодичности по угловой координате $\theta$ упрощает собственные формы.

Индексы $j$ и $m$ характеризуют формы собственных колебаний кольцевой пластины: $m$ число узловых диаметров $(m=0,1,2 \ldots), j$ - порядковый номер формы колебаний с фиксированным числом узловых диаметров $(m=1,2,3 \ldots)$. По этой причине для кольцевых пластин индекс $j$ следует заменить на двойной индекс $j m$; тогда безразмерная частота примет вид $\lambda_{j m}$, а задача колебаний кольцевых пластин становится квазиодномерной.

При $\lambda_{j m}^{2}>b$ величины $\alpha_{1 j m}$ и $\beta_{j m}$ становятся мнимыми, а модифицированные функции Бесселя первого и второго рода, содержащие их, переходят соответственно в функции Бесселя первого и второго рода.

Граничные условия и частотное уравнение. Для кольцевой пластины, колебания которой подчиняются гипотезе Тимошенко, граничные условия записываются в виде

свободный край - $\quad M_{r, j m}=0 ; M_{r \theta, j m}=0 ; Q_{r, j m}=0 ;$

шарнирное опирание - $M_{r j m}=0 ; \quad u_{j m}=0 ; \vartheta_{\theta, j m}=0 ;$

$$
\text { жесткая заделка - } \quad u_{j m}=0 ; \quad \vartheta_{r, j m}=0 ; \quad \vartheta_{\theta, j m}=0 .
$$

Для пластины, защемленной по внутреннему краю $r=r_{1}$ и свободной на внешнем крае $r=r_{2}$, должны выполняться следующие граничные условия:

$$
\begin{array}{llll}
r=r_{1} & u_{j m}=0 ; & \vartheta_{r, j m}=0 ; & \vartheta_{\theta, j m}=0 ; \\
r=r_{2} & M_{r, j m}=0 ; & M_{r \theta, j m}=0 ; & Q_{r, j m}=0 .
\end{array}
$$

Граничные условия (14) порождают систему шести линейных однородных алгебраических уравнений относительно шести произвольных постоянных:

$$
\begin{aligned}
& a_{11} C_{1 j}+a_{12} C_{2 j}+a_{13} C_{3 j}+a_{14} C_{4 j}+a_{15} C_{5 j}+a_{16} C_{6 j}=0 ; \\
& a_{21} C_{1 j}+a_{22} C_{2 j}+a_{23} C_{3 j}+a_{24} C_{4 j}+a_{25} C_{5 j}+a_{26} C_{6 j}=0 ; \\
& a_{31} C_{1 j}+a_{32} C_{2 j}+a_{33} C_{3 j}+a_{34} C_{4 j}+a_{35} C_{5 j}+a_{36} C_{6 j}=0 ; \\
& a_{41} C_{1 j}+a_{42} C_{2 j}+a_{43} C_{3 j}+a_{44} C_{4 j}+a_{45} C_{5 j}+a_{46} C_{6 j}=0 ; \\
& a_{51} C_{1 j}+a_{52} C_{2 j}+a_{53} C_{3 j}+a_{54} C_{4 j}+a_{55} C_{5 j}+a_{56} C_{6 j}=0 ; \\
& a_{61} C_{1 j}+a_{62} C_{2 j}+a_{63} C_{3 j}+a_{64} C_{4 j}+a_{65} C_{5 j}+a_{66} C_{6 j}=0 .
\end{aligned}
$$

Условие разрешимости системы (15) (равенство нулю определителя, составленного из коэффициентов при произвольных постоянных) дает частотное уравнение:

$$
\varphi\left(\lambda_{j m}\right)=\left|\begin{array}{llllll}
a_{11} & a_{12} & a_{13} & a_{14} & a_{15} & a_{16} \\
a_{21} & a_{22} & a_{23} & a_{24} & a_{25} & a_{26} \\
a_{31} & a_{32} & a_{33} & a_{34} & a_{35} & a_{36} \\
a_{41} & a_{42} & a_{43} & a_{44} & a_{45} & a_{46} \\
a_{51} & a_{52} & a_{53} & a_{54} & a_{55} & a_{56} \\
a_{61} & a_{62} & a_{63} & a_{64} & a_{65} & a_{66}
\end{array}\right|=0 .
$$

Частотное уравнение является трансцендентным уравнением и решается методом бисекции, при этом успех решения зависит от выбора шага: при малом шаге мала вероятность пропуска корней, но увеличивается время счёта. При увеличении шага возможны пропуски корней. Компьютерная система Mathematica 4.1 позволяет строить график функции $\varphi\left(\lambda_{j m}\right)$, нули которой являются корнями частотного уравнения (16), до вычисления частот и обоснованно назначить шаг в методе бисекции. При решении уравнений (15) методом бисекции шаг не должен превышать минимального расстояния между нулями функции $\varphi\left(\lambda_{j m}\right)$.

На рис. 1 представлен график функции $\varphi\left(\lambda_{j 3}\right)$.

Некоторые результаты вычислений. Вычисления, результаты которых приведены ниже, проводились для пластины с относительными размерами: $r_{1}=0.3, r_{2}=1$ и $\varepsilon=0.8$, соответствующей граничным условиям (14). В расчетах также принималось: $v=0.3$ и $k=0.86$. Проверка выполненных расчетов осуществлялась при помощи численного эксперимента - возбужде- 
ния резонансных колебаний. На рис. 2 приведен пример проверки вычисленных частот на резонанс. Проверялась частота $\lambda_{13}(j=1, m=3)$. Колебания возбуждались осевой нагрузкой, приложенной на внешней границе пластины и заданной зависимостью

$$
q=q_{0} \cos (3 \theta) \cos (\omega t),
$$

где $\omega$ - частота возбуждения; $q_{0}$ единичная перерезывающая сила, действующая на внешнем контуре пластины.

По мере приближения частоты

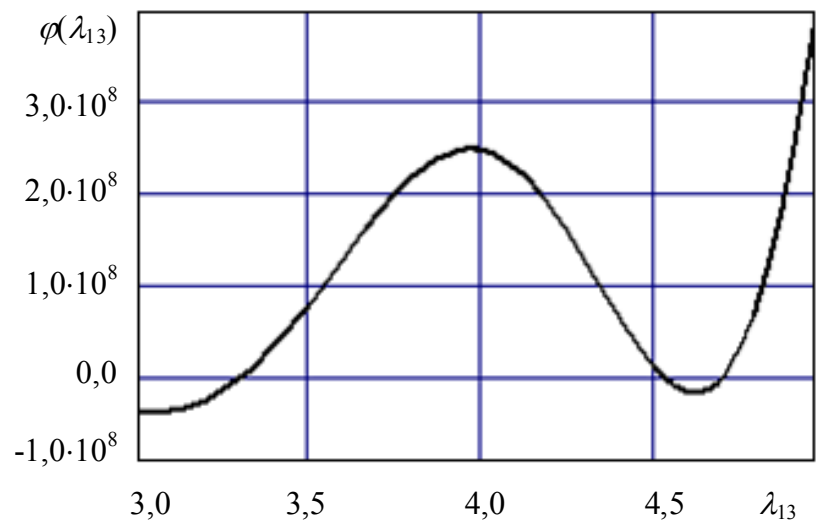

Р и с. 1. Поиск корней частотного уравнения возбуждения $\omega$ к собственной частоте $\lambda_{13}$ резко растут все параметры, в том числе вертикальные перемещения пластины $u$, зависимость которых от частоты возбуждения, приведенная на рис. 2, является типичной резонансной кривой (по оси ординат на рис. $2-u_{*}=\ln |u| \operatorname{sign}(u)$ ). Резонансные колебания возникают только при достаточно точном вычислении частот.

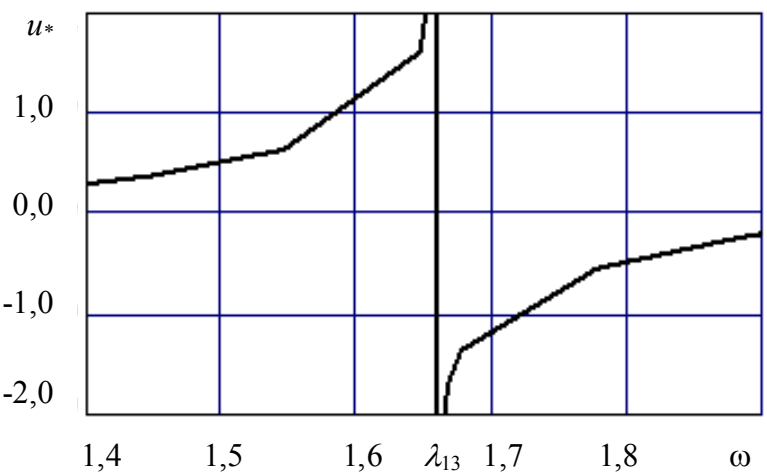

Р и с. 2. Проверка вычисленных частот на резонанс

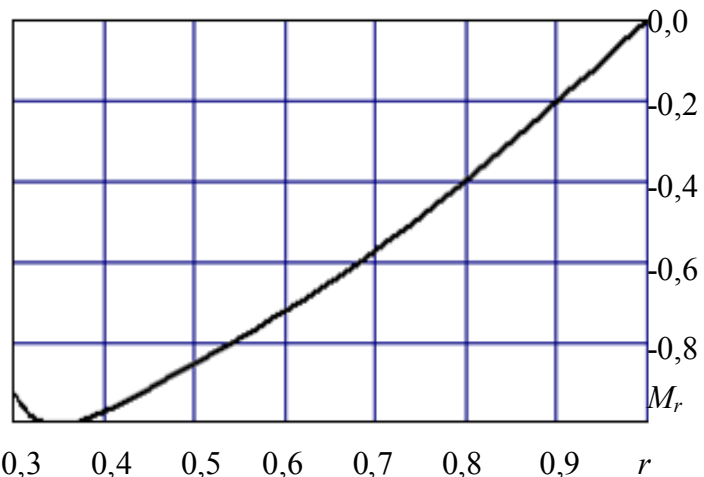

Р и с. 3. Относительные изгибающие моменты

По найденным частотам определяются формы колебаний: перемещения, осреднённые повороты, изгибающие и скручивающие моменты и перерезывающие силы, характеризующие напряженно-деформированное состояние рассматриваемой пластины в любой ее точке.

После подстановки вычисленной частоты в коэффициенты однородной системы (15) вычисляются произвольные постоянные $C_{i}(i=\overline{1,6})$ и формы колебаний. На рис. 3 приведены относительные изгибающие моменты $M_{r}$ в зависимости от радиуса $r$ пластины при $\cos (3 \theta)=1$. Вычисление относительных изгибающих и скручивающих моментов, а также перерезывающих сил позволяет определять наиболее нагруженные области элементов сооружений, схематизируемых кольцевыми пластинами.

Выводы. Разработка программ, справедливых для широкого спектра граничных условий и использующих в своем алгоритме уточнённые гипотезы теории колебаний пластин (в том числе гипотезы модели Тимошенко), способствует существенному расширению области применения последней к расчёту элементов сооружений и машин.

Построение и реализация решений в компьютерной системе Mathematica 4.1 исключает пропуски частот колебаний при вычислении частотного спектра.

\section{БИБЛИОГРАФИЧЕСКИЙ СПИСОК}

1. Кузнеиов Н.Д., Фридман Л.И., Шапошников Ю.Н. Особенности низкочастотного спектра собственных колебаний цилиндрических тел // Доклады АН СССР, 1990. 312. №1. С. 55-58.

2. Фридман Л.И., Карасева О.А. Сравнение частот прямоугольных пластин, вычисленных по уточненной и классической гипотезам // Известия вузов. Строительство. 2000. №1. С. 21-26.

3. Скучик E. Простые и сложные колебательные системы. М.: Мир, 1971. 557 с. 